\title{
Spin Structure and Critical Nucleation Frequency of Fractionalized Vortices in 2D Topologically Ordered Superfluids of Cold Atoms
}

\author{
Jun Liang Song and Fei Zhou \\ Department of Physics and Astronomy, The University of British Columbia, Vancouver, B. C., Canada V6T1Z1
}

(Dated: February 6, 2020)

\begin{abstract}
We have studied spin structures of fluctuation-driven fractionalized vortices and topological spin order in 2D nematic superfluids of cold sodium atoms. Our Monte Carlo simulations suggest a softened $\pi$-spin disclination structure in a half-quantum vortex when spin correlations are short ranged; in addition, calculations indicate that a unique non-local topological spin order emerges simultaneously as cold atoms become a superfluid below a critical temperature. We have also estimated fluctuation-dependent critical frequencies for half-quantum vortex nucleation in rotating optical traps and discussed probing these excitations in experiments.
\end{abstract}

Quantum number fractionalization has been one of the most fundamental and exciting concepts studied in modern many-body physics and topological field theories 1 , 2, 3, 4]. During the past few years, low dimensional fractionalized quantum states have further been proposed to be promising candidates for carrying out fault tolerant quantum computation [5] and their realizations in optical lattices were explored [6, 7, 8] . A closely related topic in which there has also been a growing interest is vortex fractionalization in cold gases (see for instance Ref. [9, 10, 11, 12]). Especially, motivated by experiments on low dimensional cold gases [13], Mukerjee et al studied 2D superfluids of cold atoms and analyzed the role played by fractionalized vortices in phase transitions [10]. However, spin structures of those half-quantum vortices induced by thermal fluctuations and potential topological order 14 haven't been thoroughly explored and remain to be understood. In this Letter we illustrate spin structures of fractionalized vortices; in addition, we also show that $2 \mathrm{D}$ quantum gases with short ranged spin correlations can have a topological spin order. We further estimate critical nucleation frequencies of fractionalized vortices in optical traps which can potentially be studied in experiments [15].

Our simulations illustrate that in a fundamental vortex carrying one-half of circulation quantum $h / m$ ( $h$ is the Planck constant and $m$ is the mass of atoms), there exists a softened spin disclination (i.e. a disclination in the absence of spin stiffness) even when local spin moments are strongly fluctuating at finite temperatures. The topological winding number associated with softened spin disclinations is conserved as far as the phase rigidity remains finite. This effectively leads to a non-local topological spin order. Such a nonlocal order is absent in a conventional condensate of atoms or pairs of atoms. We have further studied creation of these excitations in rotating superfluids and obtained fluctuation-dependent critical frequencies for half-quantum vortex $(H Q V)$ nucleation. $H Q V \mathrm{~s}$ in traps can be probed by measuring a precession of eigenaxes of surface quadrupole modes.

We employ the Hamiltonian introduced previously for
$\mathrm{F}=1$ sodium atoms in optical lattices 9 , 16],

$$
\begin{aligned}
\mathcal{H} & =\sum_{k} \frac{b_{L}}{2} \hat{\rho}_{k}^{2}+\frac{c_{L}}{2} \hat{\mathcal{S}}_{k}^{2} \\
& -t_{L} \sum_{<k l>}\left(\psi_{k, \alpha}^{\dagger} \psi_{l, \alpha}+\text { h.c. }\right)-\sum_{k} \mu \hat{\rho}_{k} .
\end{aligned}
$$

Here $k$ is the lattice site index and $\langle k l\rangle$ are the nearest neighbor sites. $\mu$ is the chemical potential and $t_{L}$ is the one-particle hopping amplitude. Two coupling constants are $b_{L}\left(c_{L}\right)=b(c) \frac{4 \pi \hbar^{2}}{m} \int d \mathbf{r}\left(\phi_{w}^{*}(\mathbf{r}) \phi_{w}(\mathbf{r})\right)^{2} ; b, c$ are effective s-wave scattering lengths, $\phi_{w}$ is the localized Wannier function for atoms in a periodical potential. Operators $\psi_{\alpha}^{\dagger}, \alpha=x, y, z$ create hyperfine spin-one atoms in $\frac{1}{\sqrt{2}}(|1>-|-1>),-i \frac{1}{\sqrt{2}}(|1>+|-1>)$ and $\mid 0>$ states respectively. The spin and number operators are defined as $\hat{S}_{\alpha}=-i \epsilon_{\alpha \beta \gamma} \psi_{\beta}^{\dagger} \psi_{\gamma}$, and $\hat{\rho}=\psi_{\alpha}^{\dagger} \psi_{\alpha}$. Spin correlations are mainly induced by interaction $c_{L} \hat{\mathcal{S}}^{2}$. Here we consider antiferromagnetic spin-dependent interactions such as in sodium atoms where $c_{L}>0$. Minimization of this antiferromagnetic spin-dependent interaction requires that the order parameter $\Psi_{\alpha}\left(=<\psi_{k, \alpha}^{\dagger}>\right)$ be a real vector up to a global phase, i.e. $\Psi=\sqrt{N} \mathbf{n} \exp (i \chi)$ where $\mathbf{n}$ is a unit director on a two-sphere, $\exp (i \chi)$ represents a phase director and $N$ is the number of atoms per site. All low energy degrees of freedom are characterized by configurations where $\mathbf{n}$ and $\chi$ vary slowly in space and time $[9]$. Low lying collective modes include spin-wave excitations with energy dispersion $\omega(q)=v_{s} q, v_{s}=\sqrt{c_{L} t_{L}} a$ and phasewave excitations with $\omega(q)=v_{p} q, v_{p}=\sqrt{b_{L} t_{L}} a$ (here $a$ is the lattice constant). In one-dimensions, low energy quantum fluctuations destroy spin order leading to quantum spin disordered superfluids [17]. In two-dimensions, the amplitude of quantum spin fluctuations is of order of $c_{L} / t_{L}$ and is negligible in shallow lattices as $t_{L}$ is order-ofmagnitude bigger than $c_{L}$. At finite temperatures, spin correlations are mainly driven by long wave length thermal fluctuations, analogous to quantum $1 D$ cases. This aspect was also paid attention to previously and normalsuperfluid transitions were investigated [10].

We therefore study the following Hamiltonian which effectively captures long wave length thermal fluctuations 
(a)

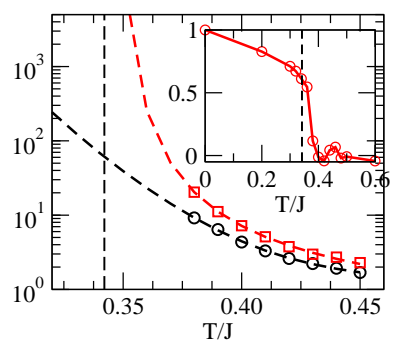

(c)

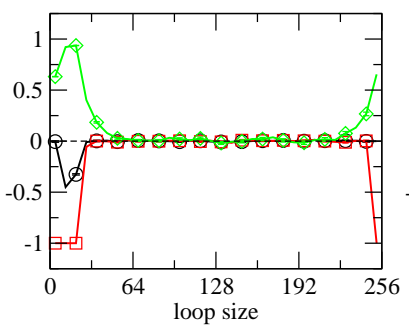

(e)

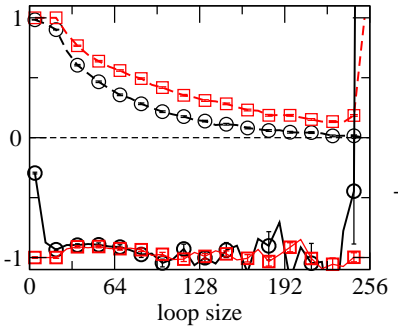

FIG. 1: (color online) a)Spin(circle) and phase(square) correlation lengths versus temperatures; inset is for the renormalized phase coupling constant $J_{p}$ (in units of $J$ ). Dashed lines are the fits to $\exp \left(A / \sqrt{T-T_{c}}\right)$ for phase correlations with $T_{c} \approx 0.35 J$ and $\exp (B / T)$ for spin correlations. b) Thermal average $\left\langle W_{s}\right\rangle$ (circle) and $\left\langle W_{p}\right\rangle$ (square) versus the loop perimeter at $T=0.33 J$ (solid line) and $T=$ $0.45 J$ (dashed line). c)-f): $\left\langle W_{s}\right\rangle_{h v}$ (circle), $\left\langle W_{p}\right\rangle_{h v}$ (square) and $\langle C\rangle_{h v}$ (diamond) averaged over configurations with $H Q V$ boundary conditions. c)-d),f) are for $T / J=0.45,0.33,0.20$ respectively; in e), we also show $\left\langle W_{s, p}\right\rangle_{h v}$ normalized in terms of back ground values $\left\langle W_{s, p}\right\rangle_{b g}$ at $T=0.33 J .\left\langle W_{s, p}\right\rangle_{b g}$ (dashed lines) are averaged over configurations with uniform phases at the boundary.

$$
H=-\sum_{<k l>} J_{k l} \mathbf{n}_{k} \cdot \mathbf{n}_{l} \boldsymbol{\Phi}_{k} \cdot \boldsymbol{\Phi}_{l}
$$

here states at each site are specified by two unit directors: a nematic director, $\mathbf{n}=\left(\cos \phi_{0} \sin \theta_{0}, \sin \phi_{0} \sin \theta_{0}, \cos \theta_{0}\right)$ and a phase director, $\boldsymbol{\Phi}=(\cos \chi, \sin \chi) . \quad J_{k l}=2 N t_{L}$ is the effective coupling between two neighboring sites and depends on $N$, the number of atoms per site. The model is invariant under the following local Ising gauge transformation: $\mathbf{n}_{i} \rightarrow s_{i} \mathbf{n}_{i}, \boldsymbol{\Phi} \rightarrow s_{i} \boldsymbol{\Phi}_{i}$, and $s_{i}= \pm 1$. In the following, we present results of our simulations on $2 \mathrm{D}$ superfluids, especially spin structures, energetics of $H Q V_{\mathrm{s}}$ and nucleation of $H Q V_{\mathrm{s}}$ in rotating optical traps using the effective Hamiltonian in Eq2.2.
$H Q V$ and underlying topological spin order Around a $H Q V$, both phase and nematic directors rotate slowly by $180^{\circ}$; in polar coordinates $(\theta, \rho)$, a $H Q V$ in condensates is represented by $\Psi(\theta, \rho)=\sqrt{N(\rho)} \exp (i \theta / 2) \mathbf{n}(\theta)$, with $\mathbf{n}=(\cos (\theta / 2), \sin (\theta / 2), 0)[12,17]$. The question here is whether, when nematic directors are not ordered, a spin disclination is still present in a $H Q V$. To fully take into account $2 D$ thermal fluctuations, we carry out Monte Carlo simulations on a square lattice of $128 \times 128$ sites and study spatial correlations between a $H Q V$ and a $\pi$ spin disclination, and topological order.

We first identify critical temperatures of the normalsuperfluid phase transition by calculating correlations and the phase rigidity. The gauge-invariant quadrupolequadrupole correlation functions we have studied are

$$
f^{s, p}\left(\mathbf{r}_{1}, \mathbf{r}_{2}\right)=<Q_{\alpha \beta}^{s, p}\left(\mathbf{r}_{1}\right) Q_{\alpha \beta}^{s, p}\left(\mathbf{r}_{2}\right)>.
$$

Here $Q_{\alpha \beta}^{s}\left(\mathbf{r}_{1}\right)=\mathbf{n}_{1, \alpha} \mathbf{n}_{1, \beta}-(1 / 3) \delta_{\alpha \beta}, \quad \alpha=x, y, z$; $Q_{\alpha \beta}^{p}\left(\mathbf{r}_{1}\right)=\boldsymbol{\Phi}_{1, \alpha} \boldsymbol{\Phi}_{1, \beta}-(1 / 2) \delta_{\alpha \beta}, \alpha=x, y$. In simulations, we have studied these correlation functions and found that the phase correlation length for $f^{p}\left(\mathbf{r}_{1}, \mathbf{r}_{2}\right)$ becomes divergent at a temperature $0.35 \mathrm{~J}$ which is identified as a critical temperature $T_{c}$. We also calculate the phase rigidity or the renormalized phase coupling $J_{p}$

$$
J_{p}=\frac{\partial^{2} F}{\partial \delta \chi^{2}}
$$

here $\delta \chi$ is a small phase difference applied across the opposite boundaries of the lattice and $F$ is the corresponding free energy. We indeed find that it approaches zero at $T_{c}$ while at $T=0 J_{p}$ takes a bare value $J$. Meanwhile, the spin correlation function $f^{s}\left(\mathbf{r}_{1}, \mathbf{r}_{2}\right)$ remains to be short ranged across $T_{c}$. By extrapolating our data to lower temperatures, we find that the spin correlation length diverges only at $T=0$ (see Fig,1a). Our simulations for correlation lengths are in agreement with previous results in Ref. [10]; they are also consistent with the continuum limit of the model in Eq2 which is equivalent to an $X Y$ model and an $O(3)$ nonlinear-sigma model.

In order to keep track of the winding of nematic directors in a wildly fluctuating back ground, we introduce the following gauge invariant $\pi$-rotation checking operator, which is essentially a product of sign-checking operators

$W_{s}=\prod_{<k l>\in \mathcal{C}} \operatorname{sign}\left(\mathbf{n}_{k} \cdot \mathbf{n}_{l}\right), W_{p}=\prod_{<k l>\in \mathcal{C}} \operatorname{sign}\left(\mathbf{\Phi}_{k} \cdot \boldsymbol{\Phi}_{l}\right) \cdot(5)$

Here the product is carried out along a closed squareshape path $\mathcal{C}$ centered at the origin of a $2 D$ lattice. $W_{s, p}$ can be either +1 or -1 ; and $W_{s}\left(W_{p}\right)$ is -1 when $\mathcal{C}$ encloses a $\pi$-spin disclination $(H Q V)$. The gauge invariant circulation of supercurrent velocity (in units of $\pi \hbar / m$ ) is defined as $C=\frac{1}{\pi} \sum_{\in \mathcal{C}} \operatorname{sign}\left(\mathbf{n}_{k} \cdot \mathbf{n}_{l}\right) \sin \left(\chi_{k}-\chi_{l}\right)$; this quantity is equal to one in a $H Q V$. 
In our simulations, we investigate the winding number $<W_{s, p}>_{h v}$ averaged over configurations where phase directors rotate by $180^{\circ}$ around the boundary of the lattice and the center plaquette. At temperatures above the normal-superfluid transition temperature $T_{c}$, both winding numbers $W_{s, p}$ and circulation $C$ are averaged to zero within our numerical accuracy(see Fig 1). And our choice of boundary conditions does not lead to a vortex or disclination configuration in the absence of phase rigidity. Below $T_{c}$, the circulation $C$ is averaged to one indicating that the boundary conditions effectively project out $H Q V$ configurations. Meanwhile, we observe loopperimeter dependent $\left\langle W_{s, p}\right\rangle_{h v}$ which can be attributed to the background fluctuations of $H Q V$ or disclination pairs. The loop-perimeter dependence of $\left\langle W_{s, p}\right\rangle_{h v}$ here is almost identical to that for uniform boundary conditions, i.e. the back ground value. After normalizing $\left\langle W_{s, p}\right\rangle_{h v}$ in terms of background winding numbers $\left\langle W_{s, p}\right\rangle_{b g}$, we find both $\frac{\left\langle W_{s}\right\rangle_{h v}}{\left\langle W_{s}\right\rangle_{b g}}$ and $\frac{\left\langle W_{p}\right\rangle_{h v}}{\left\langle W_{p}\right\rangle_{b g}}$ approach -1 (see Fig 1). We thus demonstrate that a softened disclination is spatially correlated with a $H Q V$. At the temperatures we carry out these simulations the spin correlation length is sufficiently short compared to the size of the lattice. At further lower temperatures, the spin correlation length becomes longer than the lattice size and fluctuations of pairs of disclination-anti-disclination are strongly suppressed; $\left\langle W_{s, p}\right\rangle_{h v}$ are equal to -1 for almost all loops, which corresponds to a mean field result.

Results in Fig 1 indicate that there exists a softened spin disclination in a $H Q V$. This is a distinct feature in our systems and there exist no such additional magnetic structures in $H Q V \mathrm{~s}$ in conventional molecular condensates of atom pairs discussed perviously [18]. Thus, $\pi$-disclinations like $H Q V \mathrm{~s}$ have logarithmically divergent energies and are fully suppressed in ground states. Our results also illustrate that although the average local spin quadrupole moments $Q_{\alpha \beta}^{s}$ vanish because of strong fluctuations, an overall $\pi$-rotation of nematic directors in disclinations is still conserved because of a coupling to the superfluid component. This coupling between a $H Q V$ and disclination can also be attributed [19] to a coupling between Higgs matter and discrete gauge fields [20]. Furthermore, the absence of unbound $\pi$-disclinations in superfluids indicates a topological order, similar to the one introduced previously for an isotropic phase of liquid crystal[21]. Consequently, once a conventional phase order appears below a critical temperature, a topological spin order simultaneously emerges while spin correlations remain short ranged.

The emergent topological order can be further verified by examining the average of product-operator $W_{s, p}$ over all configurations (with open boundaries). Above the normal-superfluid transition temperature $T_{c}$, we again find that $W_{s, p}$ both are averaged to zero within our numerical accuracy implying proliferation of unbound $H Q V_{\mathrm{s}}$ or disclinations. Below $T_{c}$, we study the loopperimeter dependence of average winding numbers $\left\langle W_{s, p}\right\rangle$ and find that both $\ln \left\langle W_{p}\right\rangle$ and $\ln \left\langle W_{s}\right\rangle$ are linear functions of loop-perimeter analogous to the Wilson-loop-product of deconfining gauge fields 22]; if there were unbound disclinations, one should expect that $\ln \left\langle W_{p}\right\rangle$ is proportional to, instead of the loop-perimeter, the loop-area which represents the number of unbound disclinations enclosed by the loop.

Critical frequency for $H Q V$ nucleation Let us now turn to the nucleation of those excitations in rotating traps 23, 24, 25, 26, 27, 28, 29]. To understand the critical frequency for nucleation, we study the free energy of a vortex, in a rotating frame, as a function of the distance $r$ from the axis of a cylindrical optical trap (the axis is along the $z$-direction),

$$
F_{h . v .}(r)=F_{h . v .}^{0}(r)-\Omega L_{z}(r) .
$$

Here $F_{h . v .}^{0}(r)$ is the free energy of a $H Q V$ located at distance $r$ from the trap axis in the absence of rotation, $L_{z}(r)$ is the angular momentum of the vortex state and $\Omega$ is the rotating frequency.

In a $2 D$ lattice without a trapping potential, $F_{h . v}^{0}$ is approximately equal to $\frac{\pi}{4}\left(J_{p}+J_{s}\right) \ln (L / a)$, with leading contributions from phase winding and spin twisting; here $J_{p, s}$ are renormalized phase and spin coupling respectively and $L$ is the size of system. For an integerquantum vortex $(I Q V), F_{v}^{0}$ is equal to $\pi J_{p} \ln (L / a)$. The ratio between $F_{h . v}^{0}$, and $F_{v}^{0}$ depends on the ratio $J_{s} / J_{p}$ or spin fluctuations; in the limit $L$ approaches infinity, the ratio $F_{h . v .}^{0} / F_{v}^{0}$ changes discontinuously from $\frac{1}{2}$ at $T=0$ where $J_{p} \approx J_{s}=J$ to $\frac{1}{4}$ at finite low temperatures in $2 D$ where $J_{s}$ vanishes. In simulations of a finite trap (see below), because of a finite size effect we find that this ratio varies from 0.5 to 0.2 smoothly as temperatures increase from 0 to $T_{c}$.

To study nucleation of half-quantum vortices in an optical trap, we assume a nearly harmonic trapping potential $V(r)=1 / 2 m \omega_{t r}^{2} r^{2}$, with $\omega_{t r}$ being the trap frequency. The average number of particles per site $N(r)$ has a Thomas-Fermi profile; $N(r)=N_{0}\left(1-r^{2} / R_{T F}^{2}\right)$, here $N_{0}$ is the number density at the center and $R_{T F}$ is the Thomas-Fermi radius. Furthermore, the optical lattice potential along the axial direction is sufficiently deep so that atoms are confined in a two-dimensional $x y$ plane; the in-plane lattice potential depth is set to be $5 E_{R}\left(E_{R}\right.$ is the photon recoil energy).

For the trap and lattice geometry described above, we calculate parameters in Eq 1 and obtain $t_{L}=77 \mathrm{nK}, b_{L}=$ $187 \mathrm{nK}$ and $c_{L}=10 \mathrm{nK}$. For $N_{0}=1.2$ and trap frequency $\omega_{t r}=120 \mathrm{~Hz}$, we also find that $R_{T F}=43 a$ and $L_{h . o .}=$ $4 a$ where $L_{h . o .}\left(=1 / \sqrt{2 m \omega_{t r}}\right)$ is the harmonic oscillator length. The coupling $J_{k l}$ in Eq2 depends on the distance from the center of trap and at the center, the coupling is about $154 \mathrm{nK}$. In non-rotating or slowly rotating traps, the free energy maximum is located at the center and there should be no vortices in the trap. As frequencies are increased, a local energy minimum appears at the center and becomes degenerate with the no-vortex state at a thermodynamic critical frequency (which is about 
(a)

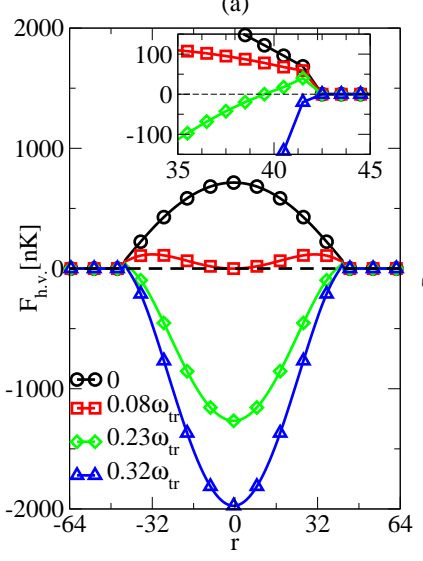

(b)

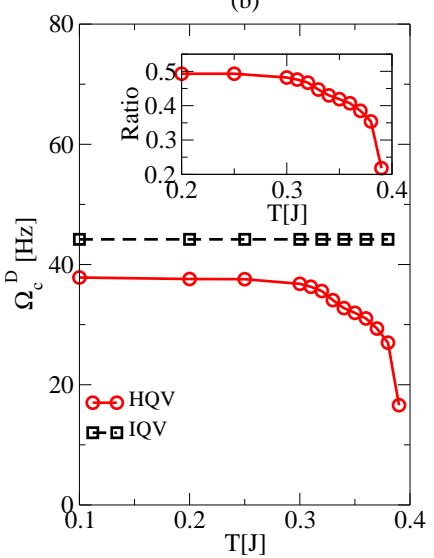

FIG. 2: (color online) a) The free energy of a $H Q V$ at a distance $r$ from the trap center at rotation frequencies $0,0.08$, 0.23 and 0.32 (in units of trap frequency $\omega_{t r}=120 \mathrm{~Hz}$ ) at $\mathrm{T}=$ $0.33 J$. Details near the edge are shown in the inset. At the center, the exchange coupling is $J=154 \mathrm{nK}$. b) The critical frequencies $\Omega_{c}^{D}$ for $H Q V \mathrm{~s}$ (solid line) and $I Q V \mathrm{~s}$ (dashed line). Inset is the temperature-dependence of the ratio between the $H Q V$ energy $F_{h . v}^{0}$ and the $I Q V$ energy $F_{v}^{0}$.

$0.08 \omega_{t r}$ at $\left.T=0.33 J\right)$; however because of a large energy barrier separating the two degenerate states as shown in Fig 2, vortices are still prohibited from entering the trap.

Further speeding up rotations results in an energetically lower and spatially narrower barrier. Within the range of temperatures studied, thermal activation turns out to be insignificant within an experimental time scale $(\sim 100 \mathrm{~ms})$ because of low attempt frequencies. So only when the spatial width of barrier becomes comparable to a hydrodynamic breakdown length [30], the barrier can no longer be felted and vortices start to penetrate into the trap. We use this criterion to numerically determine the dynamical critical frequency for vortex nucleation $\Omega_{c}^{D}$; for $I Q V \mathrm{~s}$, the calculated $\Omega_{c}^{D}$ is a flat function of $T$ (see Fig 2b) which is qualitatively consistent with earlier estimates 31].

For $H Q V \mathrm{~s}, F_{h . v}^{0}$. depends on the renormalized spin coupling $J_{s}$ and therefore the amplitude of spin fluctuations. Because of this, $\Omega_{c}^{D}$ varies from about $0.32 \omega_{t r}$ at $T=0$ where $J_{s} \approx J_{p}$ and $F_{h . v} \approx 0.5 F_{v}$ due to a finite size effect (see the inset of Fig.2), to about $0.17 \omega_{t r}$ at temperatures close to $T_{c}$ where $J_{s}=0$ and $F_{h . v} \approx 0.2 F_{v}$. In other words, this unique temperature dependence can be considered to be an indicator of fluctuation-driven vortex fractionalization. It is worth remarking that in the thermodynamic limit where $J_{s}$ approaches zero at any finite temperatures, $F_{h . v}$. approaches $\frac{1}{4} F_{v}$ as mentioned before. Consequently, $\Omega_{c}^{D}$ (about $\left.0.17 \omega_{t r}\right)$ for $H Q V_{\mathrm{s}}$ is about one-half of the critical frequency for IQVs (about $0.36 \omega_{t r}$ for the finite trap studied here). Also note that the zero temperature estimate of $\Omega_{c}^{D}$ is close to the previously obtained value of critical frequencies of $H Q V \mathrm{~s}$ in Bose-Einstein condensates 29].

The interaction between two $H Q V$ s with the same vorticity at a separation distance $d$ contains two parts. One, $V_{c c}(>0)$ is from interactions between two supercurrent velocity fields which is logarithmic as a function of $d$; and the other, $V_{s s}$ is from interaction between two spin twisting fields accompanying $H Q V \mathrm{~s}$. For a disclinationanti disclination pair, in the dilute limit one finds that $V_{c c} \sim-V_{s s}$ resulting in a cancellation of long range interactions. The resultant short-range repulsions lead to square vortex lattices found in numerical simulations 32]. For fluctuation-driven fractionalized vortices, $V_{s s}$ is almost zero and the overall interactions are always logarithmically repulsive. $H Q V$ s nucleated in a rotating trap should therefore form a usual triangular vortex lattice.

Individual vortex lines can be probed either by studying a precession of eigenaxes of surface quadrupole mode in rotating superfluids 33. In the later approach, one studies the angular momentum carried per particle in a $H Q V$ state. When a $H Q V$ is nucleated in the trap, superfluids are no longer irrotational and the angular momentum per particle is $h / 2$ rather than $h$ per particle for an integer vortex state. When a surface quadrupole oscillation across a rotating superfluid is excited, larger axes of quadrupole oscillation start to precess just as in the case of integer vortices. However, the precession rate is only one half of the value for an integer vortex state which can be studied in experiments.

In conclusion, 2D superfluids of sodium atoms have a non-local topological spin order. In rotating traps, fluctuation-driven fractionalized vortices can nucleate at a critical frequency which is about half of that for integer vortices. Observation of these exotic excitations could substantially improve our understanding of topological order and fractionalization. We thank J. Zhang and Z. C. Gu for contributions at an early stage of the project. This work is in part supported by the office of the Dean of Science, UBC, NSERC (Canada), Canadian Institute for Advanced Research, and the A. P. Sloan foundation.
[1] W. P. Su et al., Phys. Rev. B22, 2099 (1980).

[2] D. C. Tsui et al., Phys. Rev. Lett. 48, 1559 (1982).

[3] R. B. Laughlin, Phys. Rev. Lett. 50, 1395 (1983).

[4] R. Jackiw and C. Rebbi, Phys. Rev. D 13, 3398 (1976).

[5] A. Kitaev, Ann. of. Phys. 303, 2(2003); 321, 2 (2006).

[6] L. M. Duan et al., Phys. Rev. Lett. 91, 090402 (2003).

[7] H. P. Buchler et al., Phys. Rev. Lett. 95, 040402 (2005).
[8] A. Micheli et al., Nature Phys. 2, 341 (2006).

[9] E. Demler, F. Zhou, Phys. Rev. Lett. 88, 163001 (2002).

[10] S. Mukerjee et al., Phys. Rev. Lett. 97, 120406 (2006).

[11] Gordon Semenoff and Fei Zhou, Phys. Rev. Lett. 98, 100401 (2007).

[12] For general discussions, also see D. J. Thouless, Topological Quantum Numbers in Nonrelativistic Physics(World 
Scentific, 1998).

[13] Z. Hadzibabic et al., Nature 441, 1118 (2006).

[14] X. G. Wen, Quantum Theory of Many-body Systems(Oxford University Press, 2004).

[15] J. Stenger et al., Nature (London)396, 345 (1998).

[16] F. Zhou and M. Snoek, Ann. Phys. 308, 692 (2003); M. Snoek and F. Zhou, Phys. Rev. B 69, 094410 (2004).

[17] F. Zhou, Phys. Rev. Lett. 87, 080401(2001).

[18] M. W. J. Romans, Phys. Rev. Lett. 93, 020405 (2004).

[19] J. L. Song, J. Zhang and F. Zhou, unpublished.

[20] E. Fradkin and S. Shenker, Phys. Rev. D 19, 3682(1979).

[21] P. E. Lammert et al., Phys. Rev. Lett. 70, 1650(1993).

[22] K. G. Wilson, Phys. Rev. D 10, 2445(1974).

[23] K. W. Madison et al., Phys. Rev. Lett. 84, 806 (2000); K. W. Madison et al., Phys. Rev. Lett. 86, 4443 (2001).

[24] P. C. Haljan et al., Phys. Rev. Lett. 87, 210403 (2001).

[25] J. R. Abo-Shaeer et al., Science 292, 479 (2001).

[26] A. L. Fetter and A. A. Svidzinsky, J. Phys.: Condens.
Matt. 13, R135 (2001).

[27] F. Dalfovo, S. Stringari, Phys. Rev. A 63, 011601 (2000).

[28] M. Tsubota et al., Phys. Rev. A 65, 023603 (2002).

[29] T. Isoshima, K. Machida, Phys. Rev. A 66, 023602(2002).

[30] D. Feder et al., Phys. Rev. A61, 011601 (2000). The hydrodynamic breakdown length $L_{B}$ is about $\left(L_{h . o .}^{4} / 2 R_{T F}\right)^{1 / 3}$, which in our case turns out to be about $2 a$ ( $a$ is the lattice constant.).

[31] T. P. Simula et al., Phys. Rev. A66, 035601 (2002); T. Mizushima et al., Phys. Rev. A 64, 043610 (2001).

[32] Anchun Ji et al., Phys. Rev. Lett. 101, 010402(2008).

[33] F. Zambelli and S. Stringari, Phys. Rev. Lett. 81, 1754 (1998); F. Chevy et al., Phys. Rev. Lett.85, 2223 (2000). See also discussions on liquid helium in W. F. Vinen, Nature (London) 181, 1524 (1958). 